\title{
NLO QCD Corrections to Inclusive Jet and Hadron Production in DIS
}

\author{
A. Daleo, \\ Institut für Theoretische Physik, Universität Zürich, Winterthurerstrasse 190, CH-8057 Zürich, Switzerland \\ D. de Florian, and R. Sassot \\ Departamento de Física, Universidad de Buenos Aires, \\ Ciudad Universitaria, Pab.1 (1428) Buenos Aires, Argentina
}

Received on 3 December, 2006

\begin{abstract}
We analyze the order $\alpha_{s}^{2}$ corrections to the single inclusive jet and hadron cross sections in lepton-nucleon deep inelastic scattering. The full calculations are done analytically, obtaining finite NLO partonic level cross sections for these processes. We show that in both cases the dominant partonic mechanism starts at order $\alpha_{s}^{2}$, being effectively a lowest order estimate, with the consequent large factorization scale uncertainty, and the likelihood of non-negligible corrections at the subsequent order in perturbation theory.
\end{abstract}

Keywords: Semi-Inclusive DIS; Perturbative QCD

\section{INTRODUCTION}

Over the last thirty years, the DGLAP [1] approach to parton dynamics has demonstrated itself as the most adequate tool for the description of the energy scale dependence of a variety of lepton-nucleon, and nucleon-nucleon processes over a wide kinematical range. Surprisingly, not just this approximation, but the lowest order in perturbation theory within this approach (LO) gives fairly accurate estimates for paradigmatic processes such as inclusive deep inelastic scattering (DIS), provided an energy or momentum scale of a few $\mathrm{GeV}$ characterizes them. The following order (NLO) often represents small corrections, required for precise comparisons, but not for the broad picture.

In the few last years high precision DIS experiments, with a wide kinematical coverage, and the ability to measure less inclusive processes, such as those performed by the ZEUS and H1 collaborations at HERA, have extended the tests on the dynamics of partons to the limits of their kinematical reach, looking for signatures of dynamics complementary to that described by the DGLAP approach. Illustrative examples of these tests are the measurements of final state hadrons [2] and jets $[3,4]$ produced in DIS processes in the forward region, for which the LO DGLAP description fail to reproduce the data by an order of magnitude, and even NLO estimates fall short.

In a recent analysis [5], it has been shown that the striking failure of the LO description in the case of forward hadrons by no means implies the breakdown of the DGLAP dynamics, but just the inadequacy of the LO picture, which simply does not include the dominant contribution to the measured cross section: the process in which an initial state gluon is knocked out from the nucleon, and also a gluon fragments into the detected final state hadron. Indeed, the NLO approximation, which takes into account these contributions, reproduce nicely the data [5-7].

In the case of forward jets, the situation seems to be more compromised, because not only the LO estimates fail, but also NLO estimates fall short by a factor of two of the data [4]. In reference [3] this feature together with the large scale dependence of NLO calculations, has been taken as indicative of the importance of higher order corrections. In order to improve the understanding of this situation, in reference [8] we also computed the order $\alpha_{s}^{2}$ corrections to the single inclusive jet cross section in lepton-nucleon deep inelastic scattering.

The NLO corrections to single inclusive jet production in DIS have been calculated within both the phase space slicing $[9,10]$ and the subtraction formalism $[11,12]$ in order to deal with the initial and final state singularities. At variance with these previous computations, we performed a completely analytical calculation in the small cone approximation [13]. This approximation allows us to translate straightforwardly previous results on hadroproduction [5] to the case of jets, avoiding a rather cumbersome calculation and delicate numerical treatments for dealing with the collinear singularities.

Our main conclusion is that, as in the case of hadroproduction, the dominant partonic process in the most forward jet region accessed yet is the one with a gluon in the initial state and also a gluon as the main seed of the jet. This process starts at order $\alpha_{s}^{2}$, and makes the NLO effectively a lowest order estimate, with the consequent large factorization scale uncertainty, and the likelihood of non-negligible corrections at the subsequent order in perturbation.

\section{SINGLE-INCLUSIVE PROCESSES.}

In one particle inclusive processes, in addition to the parton content of the proton, which is very precisely known and parameterized in terms of parton distribution functions [14], we have to deal with the hadronization mechanism by which scattered partons develop into the observed final state hadrons. The description is completely analogous to that of the parton content: we factorize the final state collinear singularities into scale dependent fragmentation probabilities [15], ultimately measured, and retain hard contributions in a partonic cross section. Although for most observables these corrections have been explored and tested up to next to leading order accuracy and currently are being extended to NNLO, until recently there were no analytical results for NLO one particle inclusive deep inelastic scattering cross section cross section [5] 
Single inclusive jet production is similar to one particle production in the sense that we focus in the production of a single jet of particles from final state partons, and thus we expect a similar pattern of higher order corrections. Here however, there are no fragmentation functions, since partons fragment into unspecified hadrons with unit probability. This means also that we have no right to factorize final state collinear singularities into them. All final state singularities must cancel when we consider a physically meaningful jet observable. This, of course, modifies the effective scale dependence in the cross section.

From a more formal point of view, the analytic computation of higher order QCD corrections to one particle inclusive cross sections has always lagged behind that of more inclusive observables, and the reason is the much more involved singularity structure [16, 17]. As usual in these corrections, diagrams are plagued with collinear singularities that have to be identified, prescribed, and canceled to get the physical cross section, but at variance with the inclusive case, here one has to deal typically with overlapping singularities in several variables, which is a real technical challenge.

For finite transverse momentum, however it is much easier, and the argument is simple: At the lowest order in the cross section, hadrons can only be produced anti parallel to the proton, this implies that the first order contributions are finite from the collinear point of view, and the second order contributions have simple collinear singularities, otherwise they couldn't be factorized. Then, choosing the appropriate variables at partonic level the $O\left(\alpha_{s}^{2}\right)$, singularities have the same structure as in the inclusive case at $O\left(\alpha_{s}\right)$. Of course, for this same reason we have to go one order further than usual in order have a good perturbative approximation.

Specifically, the process we are considering is one in which a lepton scatters of a proton and a final state hadron is tagged.

$$
l(l)+P(P) \longrightarrow l^{\prime}\left(l^{\prime}\right)+h\left(P_{h}\right)+X,
$$

The cross section will be given by a convolution of a parton density, a fragmentation function, and the partonic level cross section:

$$
\begin{aligned}
\frac{d \sigma^{h}}{d x_{B} d Q^{2}}=\sum_{i, j, n} & \int_{0}^{1} d \xi \int_{0}^{1} d \zeta \int \mathrm{dPS}^{(n)} \\
& {\left[f_{i}(\xi) D_{h / j}(\zeta) \frac{d \sigma_{i j}^{(n)}}{d x_{B} d Q^{2} \mathrm{dPS}^{(n)}}\right] . }
\end{aligned}
$$

where $\sigma_{i j}^{(n)}$ is the partonic level cross section corresponding to the process

$$
l(l)+i\left(p_{i}\right) \longrightarrow l^{\prime}\left(l^{\prime}\right)+j\left(k_{j}\right)+\mathrm{n}-1 \text { additional partons },
$$

before renormalization of the coupling constant and factorization of collinear singularities. $f_{i}(\xi)$ and $D_{h / j}(\zeta)$ are the bare parton densities and fragmentation functions, and $\operatorname{dPS}^{(n)}$ the $n$-parton phase space. $\xi$ is the proton momentum fraction carried by the parton $i$ and $\zeta$ is the fraction of parton $j$ momentum taken away by the final state hadron. In addition, we define the usual DIS variables,

$$
\begin{aligned}
Q^{2} & =-q^{2}=-\left(l^{\prime}-l\right)^{2}, & x_{B} & =\frac{Q^{2}}{2 P \cdot q}, \\
y_{e} & =\frac{P \cdot q}{P \cdot l}, & S_{H} & =(P+l)^{2} .
\end{aligned}
$$

Integrating the cross section over the spectator partons and prescribing the singularities we obtain a cross section that has the same singularity structure as in inclusive DIS at $O\left(\alpha_{s}\right)$ :

$$
\begin{aligned}
\frac{d \sigma_{i j}^{(2)}}{d x_{B} d Q^{2} d y d z}= & \frac{c_{q} C_{\varepsilon}^{2}}{\xi x_{B}^{2} S_{H}^{2}} \\
& \left\{\frac{1}{\varepsilon} \mathcal{P}_{1 i j}^{(2)}(\rho, y, z)+C_{i j}^{(2)}(\rho, y, z)+O(\varepsilon)\right\},
\end{aligned}
$$

The standard NLO definitions for parton densities and fragmentation functions automatically cancel these remaining singularities. Of course, the coefficients are rather long because they include the finite terms of $O\left(\alpha_{s}^{2}\right)$, which only show up at NNLO in the inclusive case.

At NLO, which for this processes is $O\left(\alpha_{s}^{2}\right)$ in the cross section, we have the following real and virtual contributions initiated by either a quark or a gluon, and any of the final state partons can be attached to a fragmentation function, one at a time.

$$
\begin{aligned}
& \text { Real contributions } \begin{cases}\gamma+q(\bar{q}) & \rightarrow g+g+q(\bar{q}) \\
\gamma+q_{i}\left(\bar{q}_{i}\right) & \rightarrow q_{i}\left(\bar{q}_{i}\right)+q_{j}+\bar{q}_{j} \\
\gamma+q_{i}\left(\bar{q}_{i}\right) & \rightarrow q_{i}\left(\bar{q}_{i}\right)+q_{i}+\bar{q}_{i} \\
\gamma+g & \rightarrow g+q+\bar{q}\end{cases} \\
& \text { Virtual contributions } \begin{cases}\gamma+q(\bar{q}) & \rightarrow q(\bar{q}) \\
\gamma+q(\bar{q}) & \rightarrow g+q(\bar{q}) \\
\gamma+g & \rightarrow q+\bar{q}\end{cases}
\end{aligned}
$$

Notice that compared to the previous order, we have new channels, for example the one in which a gluon initiates the process and also a gluon fragments, corresponding to the diagrams in Fig. 1
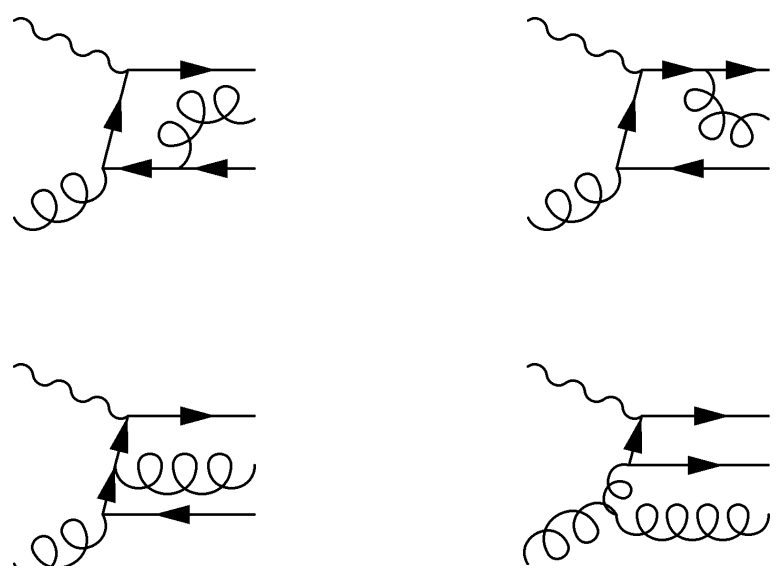

FIG. 1: Gluon initiated contributions at $O\left(\alpha_{s}^{2}\right)$ 


\section{PHENOMENOLOGICAL IMPLICATIONS}

The obvious question at this point is if computing all the $O\left(\alpha_{s}^{2}\right)$ contributions are worth the effort. The answer should be found in $\mathrm{K}$-factors, which are defined as the ratio between the NLO and the LO predictions. And the answer seems to be negative, as shown in Fig. 2, because the K-factor as a function of transverse momentum for a photon virtuality of $200 \mathrm{GeV}^{2}$ in a wide range Bjorken momentum fractions is, as usual, less than a $20 \%$ effect far from the factor of four we need to match the data.

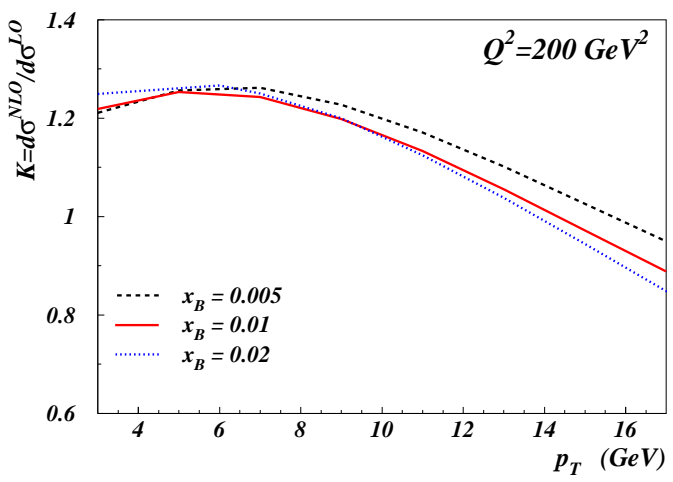

FIG. 2: K-factor as a function of $p_{T}$.

We still have the arbitrariness in choice for the renormalization and factorization scale. The standard choice would be to take some combination between the transverse momentum and the virtuality of the photon, which are most conspicuous scales in the problem, i.e.

$$
\mu_{0}^{2}=\frac{\left(Q^{2}+p_{T}^{2}\right)}{2} .
$$

Varying this scale as much as a factor of ten around the standard choice the cross section varies but not enough to explain H1 data, as shown in Fig. 3.

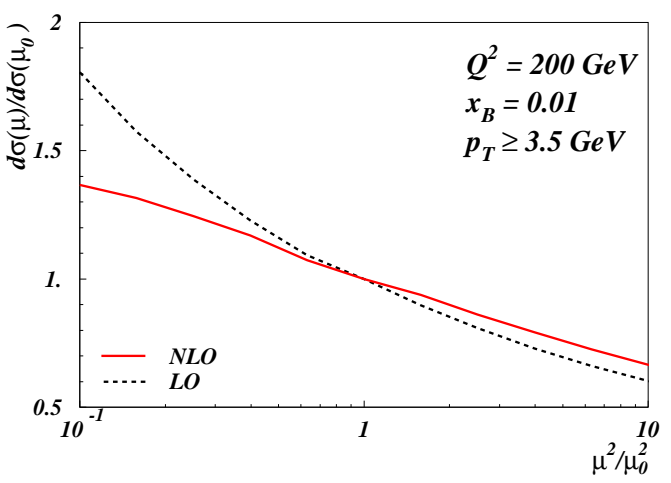

FIG. 3: K-factor as a function of the scale.

It is worthwhile at this point to have a closer look at the data, which correspond to neutral pions produced in positron proton DIS processes in these rather stringent cuts on the angle and pion energy fractions [2]. As a function of Bjorken momentum fraction, the measured cross section looks as shown in Fig. 4 in different $Q^{2}$ bins. The LO prediction badly underestimates the data, however when we switch on NLO corrections, the situation changes dramatically: NLO corrections are huge.

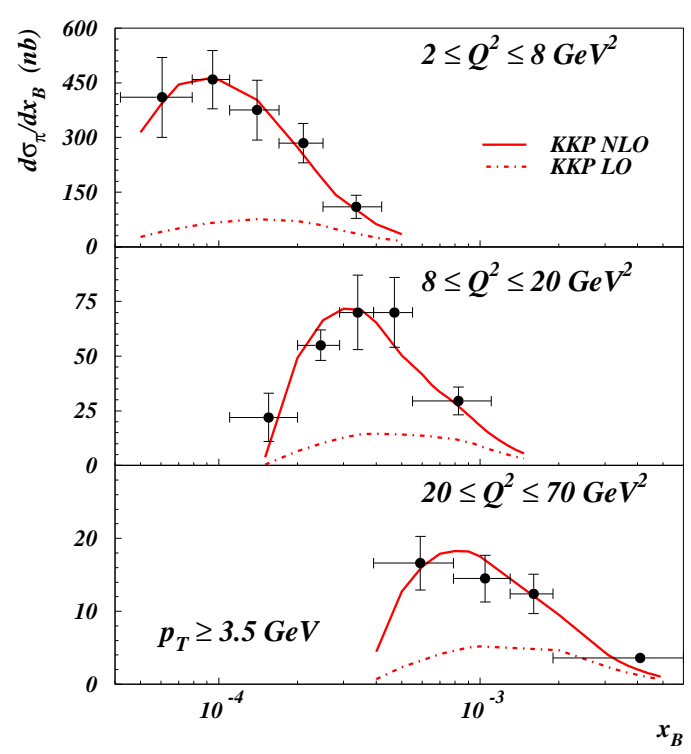

FIG. 4: NLO cross sections, against H1 data [2]

But what is so special about this observable, that makes so important the corrections, even if they were not in the $\mathrm{K}$ factors shown in Figs. 2 and 3? The answer is the kinematical cuts. In Fig. 5 we see again the K-factor as a function of Bjorken $\mathrm{x}$ with and without $\mathrm{H} 1$ cuts on angle and energy fractions. Applying cuts, the $\mathrm{K}$-factors can become as large

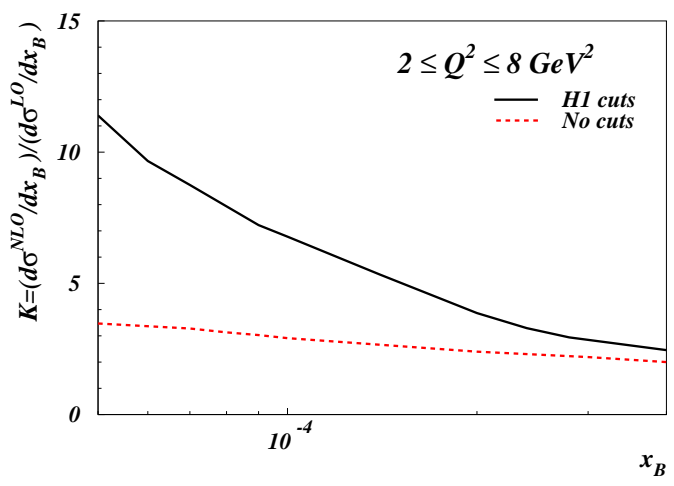

FIG. 5: K-factors with and without cuts

as a factor ten. This usually means that a new channel has been open, a process that was not present at a given order is present at the following, and in this case enhanced by a particular kinematical cut. This channel happens to be precisely the 
gluon to gluon process. In Fig. 6 here we have the LO contribution as a white line and the different NLO contributions discriminated.

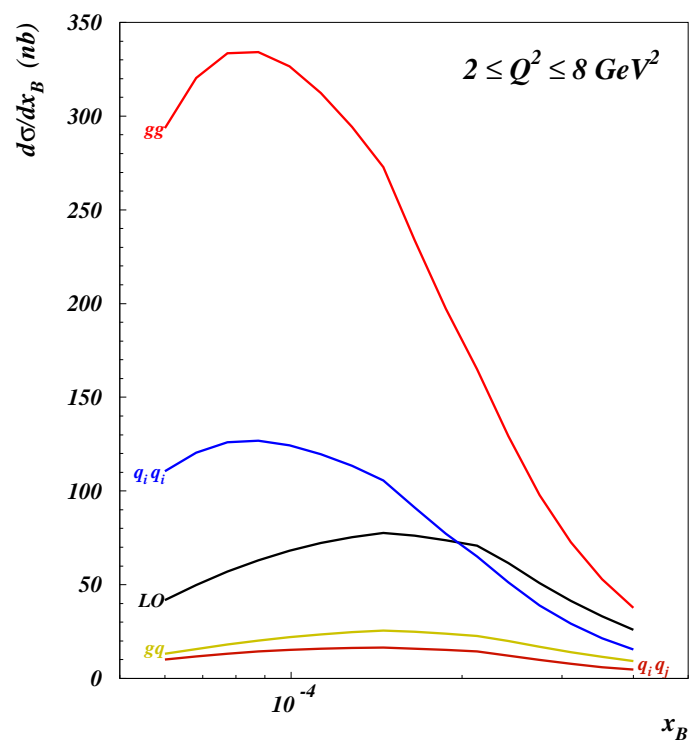

FIG. 6: Contributions to the cross section

In the case of the NLO jet cross section again the most striking feature is the growth of the NLO corrections as the rapidity of the jets increases. In Fig. III we plot the cross section in three regions of rapidity as a function of transverse jet momentum. We include the data of H1 [18] for comparison. NLO corrections, which are moderate for central rapidities, become significantly large in the forward region.

In Fig. 8 we show the different partonic contributions in the three rapidity regions. While in the central region the LO contribution is very close to the full NLO estimate, in the forward region it is significantly smaller. In the former the cross section is dominated by the $\sigma_{g \rightarrow q}$ contributions, which are already present at $\mathrm{LO}$, while in the latter the dominants are $\sigma_{g \rightarrow g}$, which are pure NLO.

Since the dominant partonic process in the forward region is accounted at NLO for the first time, it is effectively a LO estimate and most probably receives significant higher order corrections. The first round of corrections for other partonic processes in this kinematic region rise typically to $50 \%$ ef- fects, so it would not be surprising that the NLO estimate falls short of the data, specially if a more stringent kinematic range is explored. This is precisely what the ZEUS collaboration has reported in preliminary analyses of measurements in the very forward region [19].

In Fig. 9 we plot the NLO estimates as distributions in different variables together with ZEUS preliminary data for rapidities between 2.0 and 3.5 [19]. The NLO estimate falls short of the data, and only allowing a rather large scale uncertainty it may be considered consistent with the measurements, specially at small $x_{B}$.

Again the $\sigma_{g \rightarrow g}$ contributions dominate the cross section, specially at low $x_{B}$ where the gluon parton density grows dramatically and in the middle of the rapidity range, as shown in Fig. 10. In these two regions one can expect the first order corrections to these processes, starting at NNLO, to be significant. In fact, it is there where the NLO estimate can be more distant to the data with a particular choice for the scale, and where NNLO corrections became really necessary.

\section{CONCLUSIONS}

We have computed the single inclusive jet and hadron deep inelastic scattering cross section at $O\left(\alpha_{s}^{2}\right)$. In both cases we have found that the dominant partonic processes in the very forward region start at order $\alpha_{s}^{2}$, being effectively a lowest order estimate. As in any lowest order calculation, there is a large factorization scale uncertainty which can not be neglected, and it is likely that there will be large corrections at the subsequent order in perturbation. Rather than a breakdown of the DGLAP approach, these processes seem to open a window to NNLO. This feature is expected to be even more apparent at higher rapidities, and the corresponding measurements will constitute an obligatory benchmark for the study of QCD at NNLO.

\section{Acknowledgments}

Partially supported by CONICET, Fundación Antorchas, UBACYT and ANPCyT, Argentina. R.S. warmly acknowledges M. B. Gay Ducati, and the Local Comitee of the I Latin American workshop on High Energy Phenomenology for their hospitality during the meeting where this talk was presented.
[1] V. N. Gribov, L. N. Lipatov, Yad. Fiz. 15, 781 (1972) and 1218 [Sov. J. Nucl. Phys. 15, 438 and 675 (1972)];

Y. L. Dokshitzer, Sov. Phys. JETP 46, 641 (1977) [Zh.Eksp.Teor.Fiz. 73, 1216 (1977)];

G. Altarelli and G. Parisi, Nucl. Phys. B 126, 298 (1977).

[2] A. Aktas et al. [H1 Collaboration], Eur. Phys. J. C 36, 441 (2004) [arXiv:hep-ex/0404009].

[3] S. Chekanov et al. [ZEUS Collaboration], hep-ex/0502029.

[4] A. Aktas et al. [H1 Collaboration], hep-ex/0508055.
[5] A. Daleo, D. de Florian, and R. Sassot, Phys. Rev. D 71, 034013 (2005) [arXiv:hep-ph/0411212].

[6] B. A. Kniehl, G. Kramer, and M. Maniatis, Nucl. Phys. B 711, 345 (2005) [Erratum-ibid. B 720, 345 (2005)]

[7] P. Aurenche, R. Basu, M. Fontannaz, and R. M. Godbole, Eur. Phys. J. C 34, 277 (2004). [arXiv:hep-ph/0312359].

[8] A. Daleo and R. Sassot, arXiv:hep-ph/0511189.

[9] E. Mirkes and D. Zeppenfeld, Phys. Rev. Lett. 78, 428 (1997) [arXiv:hep-ph/9609231]. 


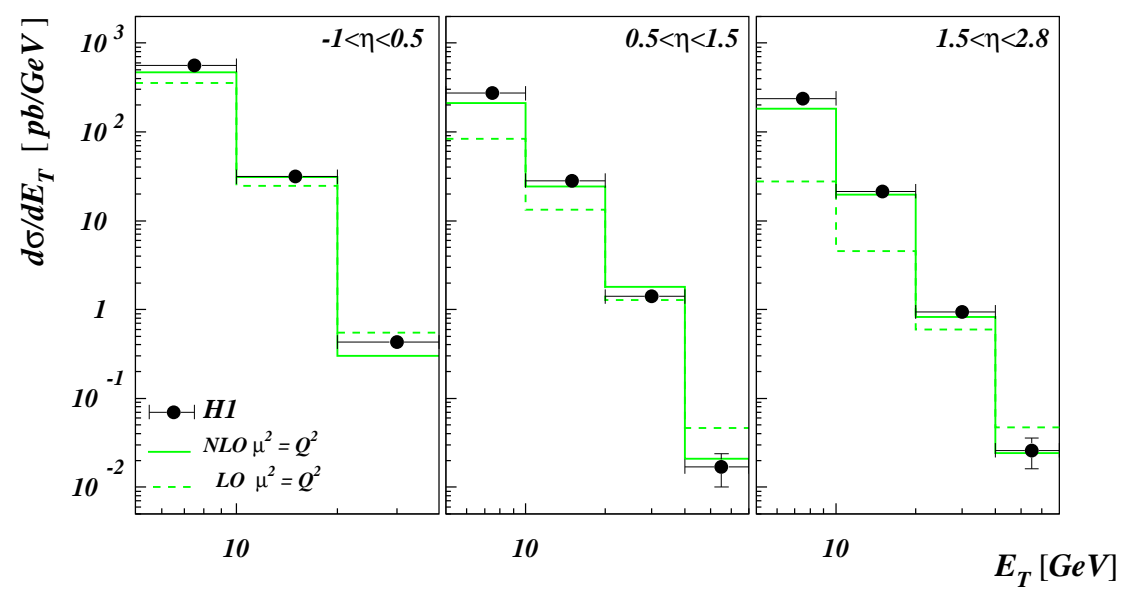

FIG. 7: Jet cross sections in H1 kinematics
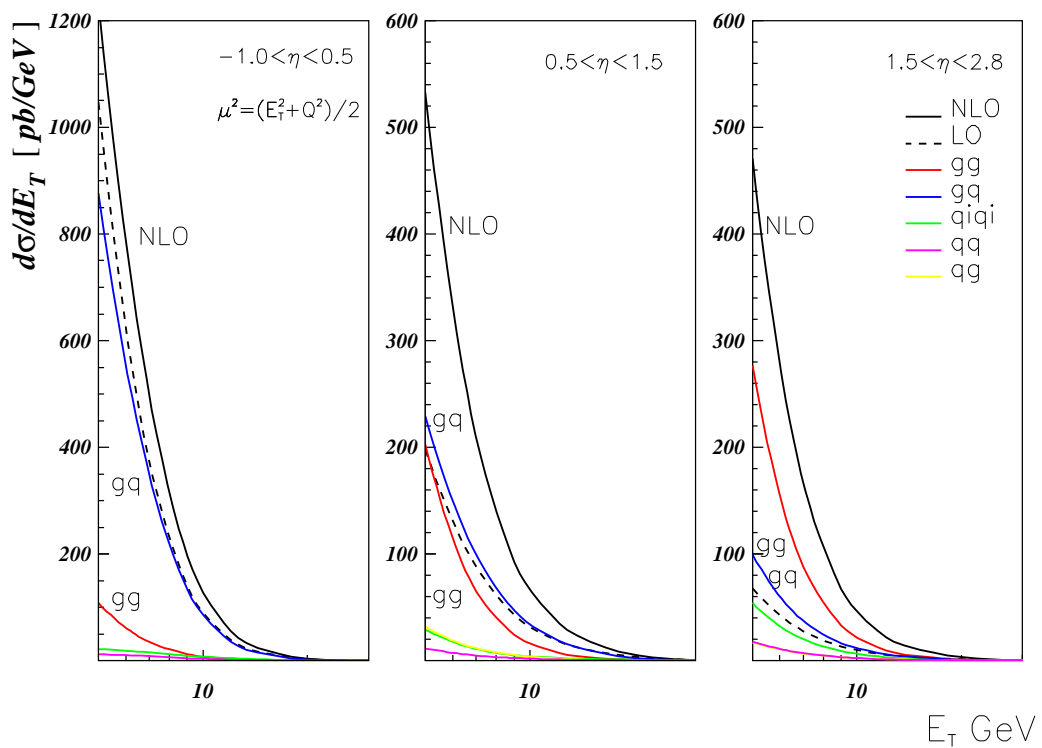

FIG. 8: Contributions to the jet cross section

[10] G. Kramer and B. Potter, Eur. Phys. J. C 5, 665 (1998) [arXiv:hep-ph/9804352].

[11] S. Catani and M. H. Seymour, Phys. Lett. B 387, 287 (1996); S. Catani and M. H. Seymour, Nucl. Phys. B 485, 291 (1997) [Erratum-ibid. B 510, 503 (1997)].

[12] D. Graudenz, arXiv:hep-ph/9710244.

[13] G. Sterman and S. Weinberg, Phys. Rev. Lett. 39, 1436 (1977), M. A. Furman, Nucl. Phys. B 197, 413 (1981).

[14] A. D. Martin, R. G. Roberts, W. J. Stirling, and R. S. Thorne, Eur. Phys. J. C 28, 455 (2003) [arXiv:hep-ph/02112080].

[15] B. A. Kniehl, G. Kramer, and B. Potter, Nucl. Phys. B 582, 514
(2000) [arXiv:hep-ph/0010289].

[16] A. Daleo and R. Sassot, Nucl. Phys. B 673, 357 (2003) [arXiv:hep-ph/0309073].

[17] A. Daleo, C. A. Garcia Canal, and R. Sassot, Nucl. Phys. B 662, 334 (2003) [arXiv:hep-ph/0303199].

[18] C. Adloff et al. [H1 Collaboration], Phys. Lett. B 542, 193 (2002) [arXiv:hep-ex/0206029].

[19] S. Chekanov et al. [ZEUS Collaboration], Contributed paper N370 to the HEP2005 International Europhysics Conference on High Energy Physics, July 21st-27th, 2005, Lisbon, Portugal. 

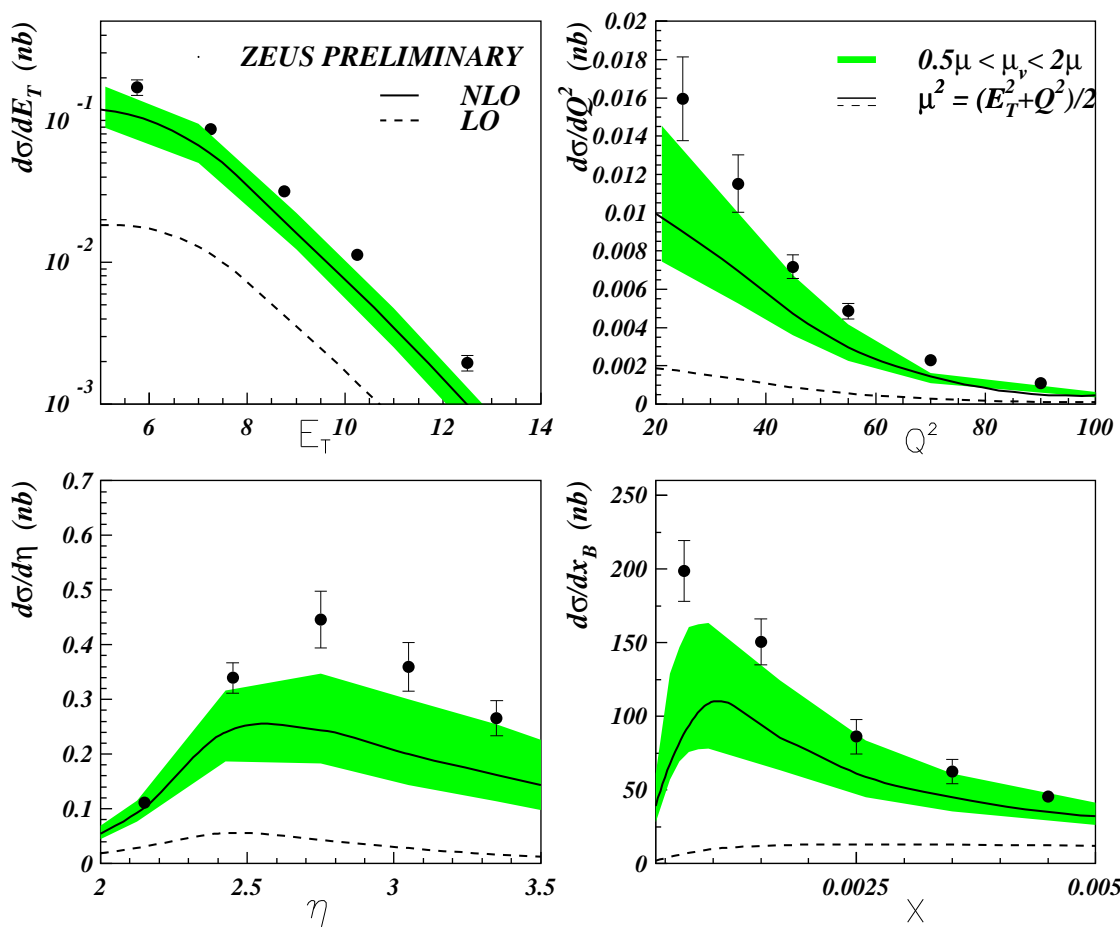

FIG. 9: Jet cross section and Zeus preliminary data
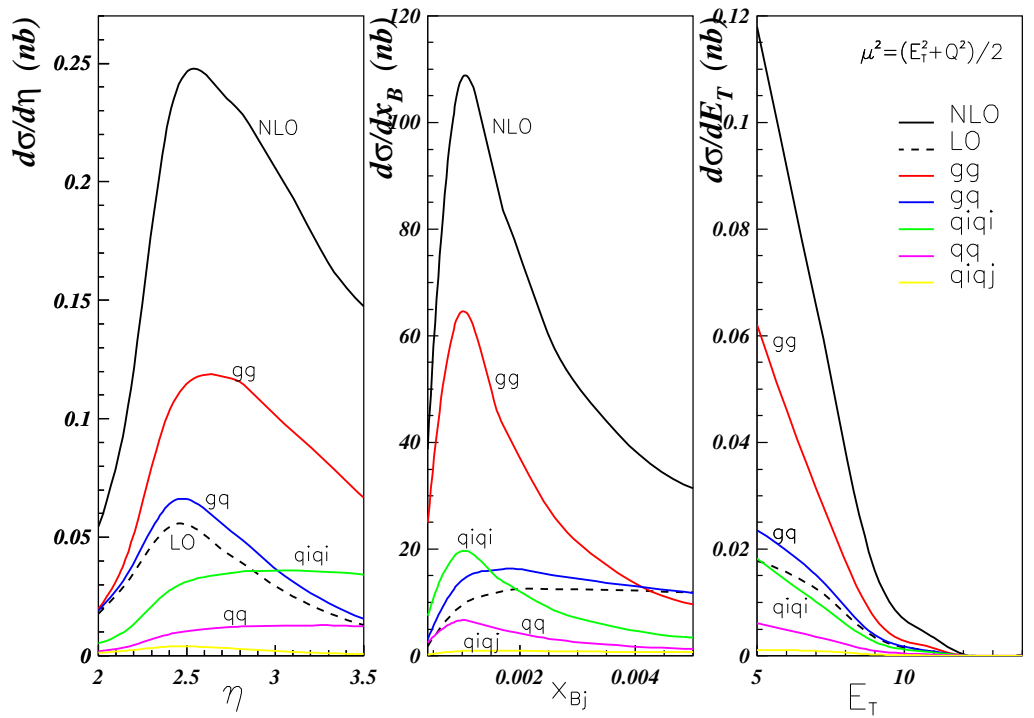

FIG. 10: Partonic contributions to the jet cross section 